\title{
Herniação Meningoencefálica do Osso Temporal
}

\section{Temporal Bone Meningoencephalic Herniation}

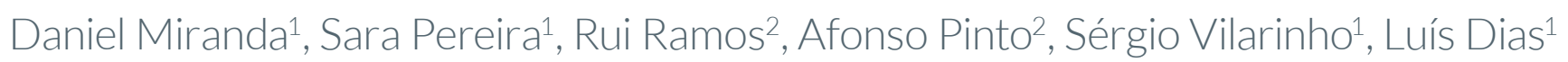

\section{RESUMO}

INTRODUÇÃO: As herniações meningoencefálicas do osso temporal correspondem à presença de parênquima encefálico e/ou meninges prolapsados através de um defeito ósseo temporal. Trata-se de uma condição rara, podendo ter consequências catastróficas para o paciente.

DESCRIÇÃO DE CASO: Doente de 69 anos, sexo feminino, com antecedentes pessoais de otite média crónica, submetida a cirurgia otológica, hipertensão arterial e neoplasia da mama. Admitida no serviço de urgência por convulsões tónico-clónico generalizadas secundárias a meningoencefalite com ponto de partida em foco infecioso otológico. A investigação imagiológica revelou a presença de uma herniação meningoencefálica, que foi corrigida cirurgicamente por equipa multidisciplinar de Otorrinolaringologia e Neurocirurgia.

DISCUSSÃO/CONCLUSÕES: O tratamento cirúrgico tem por objetivo a remoção de tecidos meningoencefálicos herniados, reparação do defeito ósseo e recuperação da audição. O tamanho e localização do defeito ósseo e da herniação meningoencefálica são fatores decisivos na escolha da via de abordagem cirúrgica.

PALAVRAS-CHAVE: Encefalocele; Hérnia; Meningocele; Osso Temporal

\section{ABSTRACT}

BACKGROUND: Temporal bone meningoencephalic herniations correspond to the presence of brain parenchyma and/or prolapsed meninges through a bone defect. It is a rare condition with potentially catastrophic consequences for the patient. CASE DESCRIPTION: Sixty-nine year-old female patient with past medical history of chronic otitis media submitted to otologic surgery, arterial hypertension and breast cancer. Admitted to emergency department with tonic-clonic seizures secondary to meningoencephalitis triggered by otologic infection. The imaging investigation revealed the presence of a meningoencephalic herniation that was surgically treated by a multidisciplinary ENT and neurosurgery team. 
DISCUSSION/CONCLUSIONS: Surgical treatment aims to remove meningoencephalic herniated tissue, bone defect repair and hearing recovery. Size and location of bone defect and meningoencephalic herniation are decisive factors in surgical approaches choice.

KEYWORDS: Encephalocele; Hernia; Meningocele; Temporal Bone

\section{INTRODUÇÃO}

As herniações meningoencefálicas (HME) do osso temporal correspondem à presença de tecidos meníngeos e/ou parênquima cerebral prolapsados no ouvido médio através de um defeito ósseo temporal. ${ }^{1}$ Trata-se de uma entidade rara e com potencial de complicações fatais para o paciente, descrita pela primeira vez em 1902 por Caboche. ${ }^{2}$

No que respeita à nomenclatura desta patologia, alguns autores preferem o termo herniação meningoencefálica ao invés de meningoencefalocelo, uma vez que a primeira designação descreve a presença de tecido meníngeo e/ou cerebral herniado e exclui o termo "celo", tendo em conta que a presença de uma área quística, contendo líquido cefalorraquidiano (LCR), não é um achado constante. ${ }^{3}$

Para a ocorrência de uma HME é necessária a presença de um defeito ósseo temporal cuja etiologia pode ser devida a: otite média crónica (OMC), simples ou colesteatomatosa, traumatismo temporal, cirurgia otológica ou irradiação prévias, neoplasia, defeito congénito da base do crânio ou idiopática. ${ }^{1,4}$

Os autores descrevem um caso clínico de uma HME do osso temporal numa doente com antecedentes de cirurgia otológica prévia e cuja apresentação clínica foi um estado de mal epiléptico secundário a meningoencefalite de causa otogénica. A doente foi submetida a correção cirúrgica por via transmastoideia com cura da HME.

\section{DESCRIÇÃO DO CASO}

Doente de 69 anos, sexo feminino, com antecedentes pessoais de hipertensão arterial, neoplasia da mama submetida a mastectomia e quimioterapia e OMC colesteatomatosa esquerda submetida a mastoidectomia canal wall-up e timpanoplastia tipo I há 20 anos. Iniciou quadro de otalgia e otorreia esquerdas associadas a cefaleias holocranianas. Posteriormente apresentou alteração do estado de consciência com desenvolvimento de convulsões tónico-clónico generalizadas, pelo que, foi encaminhada ao serviço de urgência (SU). Foi admitida na sala de emergência em coma e respiração ineficaz que foi revertida com entubação orotraqueal. Na avaliação inicial realizou tomografia computorizada (TC) crânio- -encefálica (CE) que revelou ocupação da caixa timpânica e mastoide esquerda por material de tecidos moles, sugerindo processo inflamatório, associado a lesão parenquimatosa cerebral temporo-basal sugestiva de processo de cerebrite. Foi também realizada punção lombar (PL) com saída de liquor turvo, cujo exame citoquímico revelou pleocitose com predomínio de polimorfonucleares, proteinorráquia e glicorráquia. A doente foi avaliada por Otorrinolaringologia (ORL) no SU que constatou, à otoscopia, presença de otorreia no canal auditivo ex-

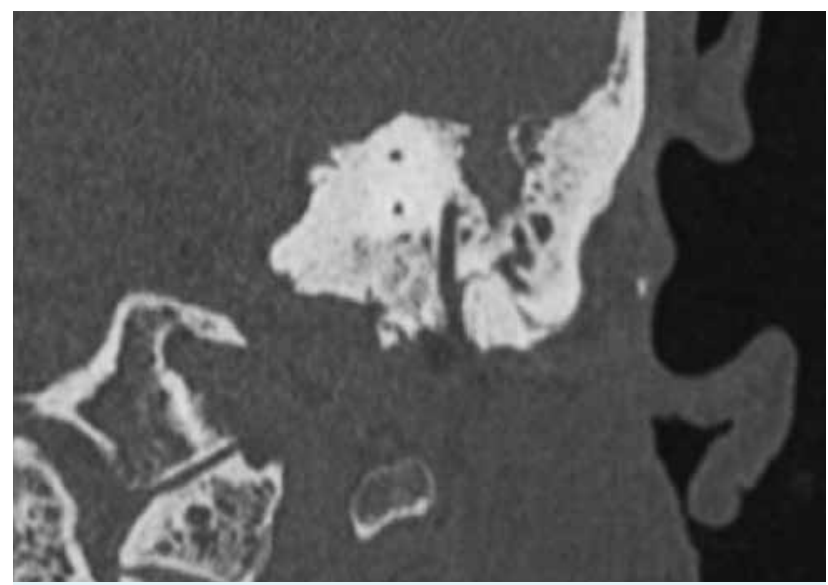

FIGURA 1. Tomografia computorizada de ouvidos.

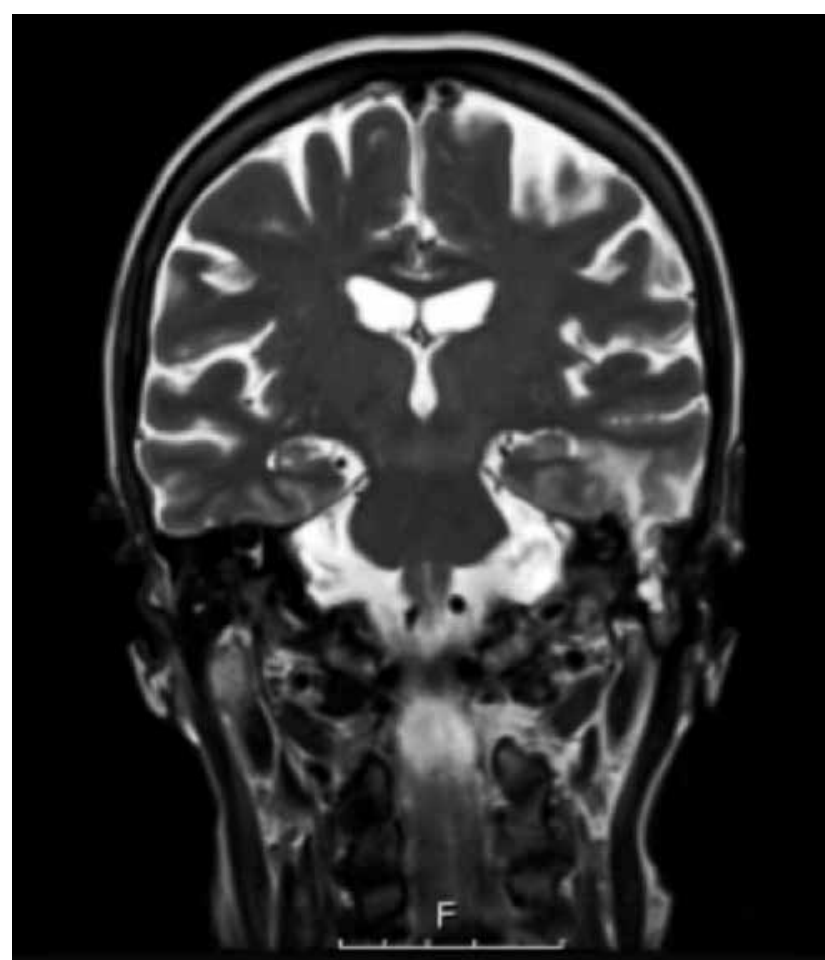

FIGURA 2. Ressonância magnética crânio-encefálica pré-operatória. 

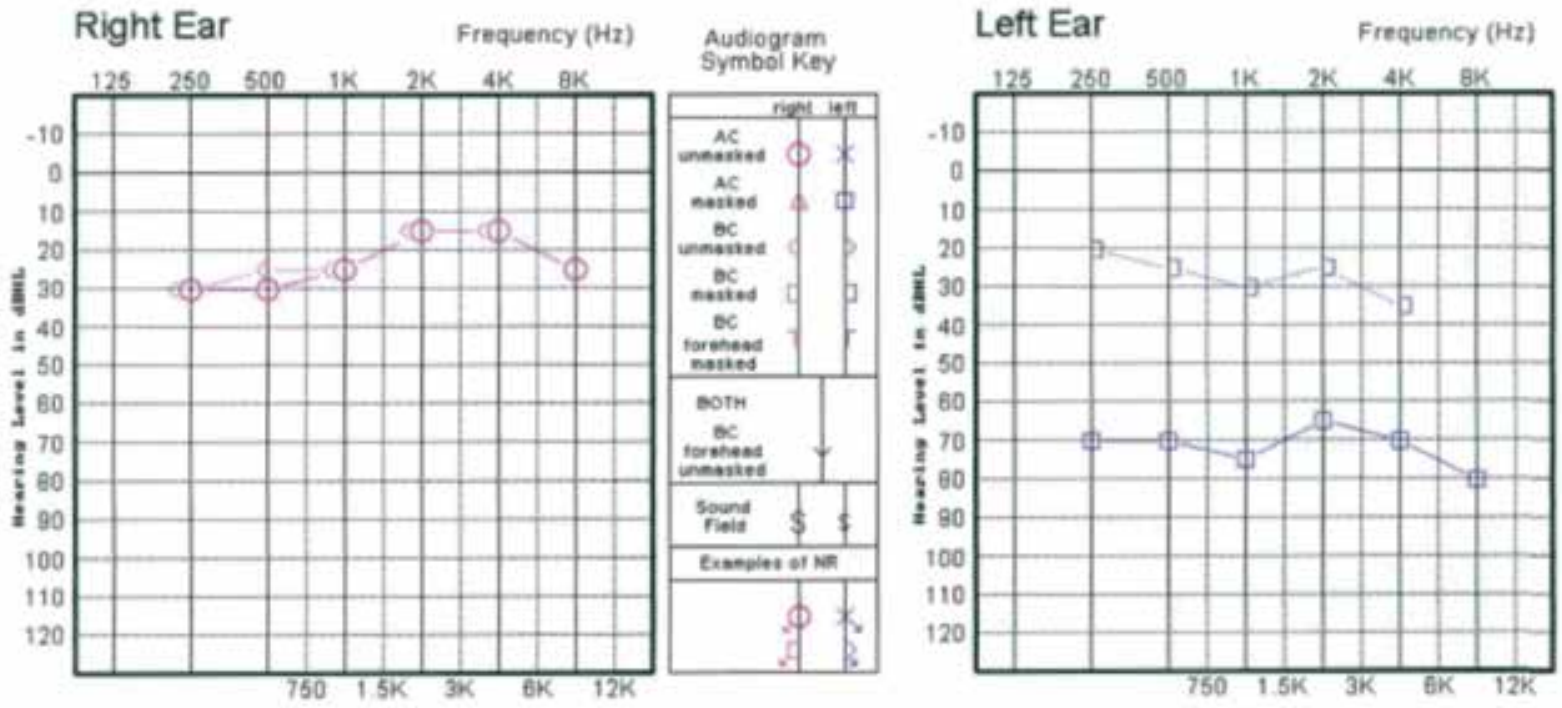

FIGURA 3. Audiograma tonal pré-operatório.
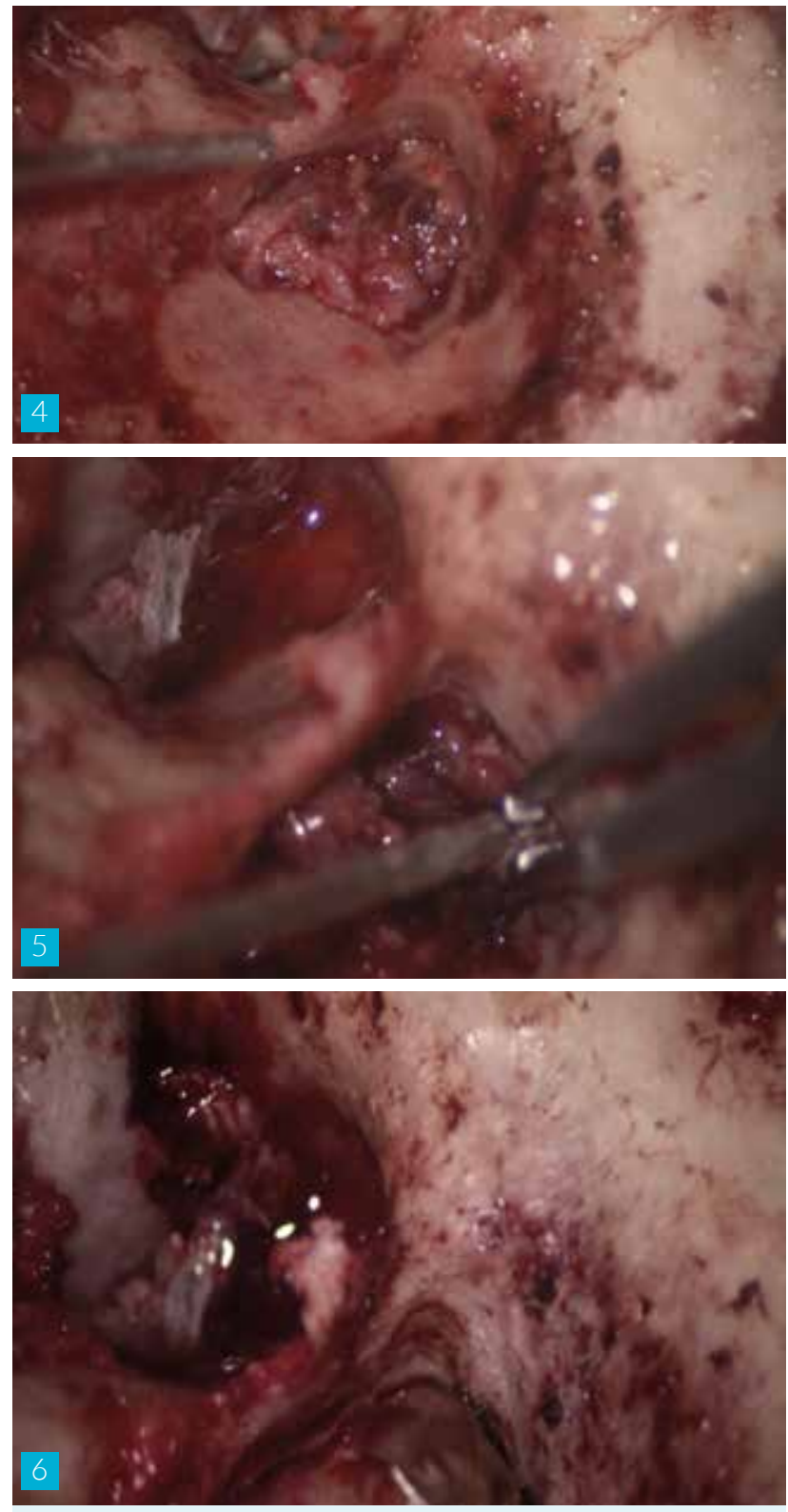

FIGURAS 4 A 6. Aspetos intra-operatórios.

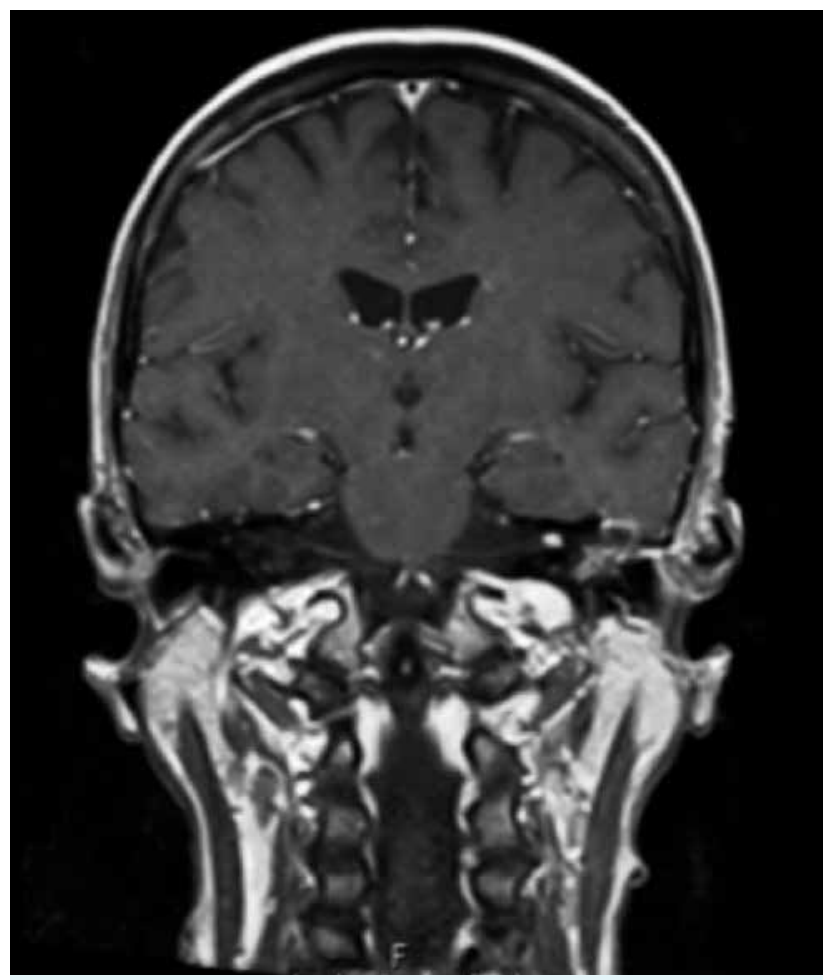

FIGURA 7. Ressonância magnética crânio-encefálica pós-operatória.

terno com membrana timpânica espessada e exsudativa. Foi colhido exsudado auricular e liquor para exame microbiológico, assim como, hemoculturas. A doente foi então admitida na unidade de cuidados intensivos (UCI) com o diagnóstico de meningoencefalite associada a cerebrite temporal com ponto de partida em foco infecioso otológico. Iniciou antibioticoterapia empírica com vancomicina e meropenem, permanecendo sedada e ventilada, com estabilidade hemodinâmica. Foi extubada ao $2^{\circ}$ dia de internamento na $\mathrm{UCl}$, tendo alta desta unidade ao fim de 4 dias. Foi então admitida na enfermaria de Neurologia para continuação de cuidados. 
Os exames microbiológicos do exsudado auricular e liquor foram positivos para Streptococcus pneumoniae multissensível, pelo que foi modificado o esquema de antibioticoterapia inicial, com suspensão da vancomicina e manutenção do meropenem.

A doente foi novamente avaliada por ORL, apresentando à otoscopia, tímpanos íntegros, com alterações esclero-cicatriciais à esquerda, sem evidência de otorreia ou matriz de colesteatoma. Foi requisitada TC de ouvidos que revelou ocupação da mastoide e caixa timpânica com material de tecidos moles associada a solução de continuidade do tegmen mastoideu (Fig. 1). Foi também requisitada ressonância magnética crânio-encefálica (RM CE) que revelou foco de cerebrite temporal, sem coleção abcedada, associado a herniação meningoencefálica temporal esquerda (Fig. 2). Os exames audiométricos revelaram hipoacusia mista esquerda com intervalo aéreo-ósseo de aproximadamente 40 dB. (Fig. 3). A doente foi proposta para correção cirúrgica da HME por equipa multidisciplinar composta por ORL e Neurocirurgia. Durante a cirurgia, após mastoidectomia, foi identificada a presença de parênquima cerebral herniado através de feito ósseo e dural. Procedeu-se à cauterização e secção do tecido desvitalizado, sendo o defeito corrigido através da interposição de dura liofilizada (TissuDura ${ }^{\circledR}$ ) e fáscia temporalis fixadas com cola de fibrina (Figs. 4 a 6). A doente manteve antibioticoterapia durante 30 dias, tendo tido alta clínica ao final de 44 dias de internamento.

Observada em consulta de seguimento, após 3 meses, apresentando-se assintomática e sem evidência de lesões retrotimpânicas à otoscopia. Realizou RM CE de controlo sem evidência de recidiva da HME (Fig. 7). No audiograma tonal pós-operatório mantinha hipoacusia mista esquerda, sem modificação significativa em relação ao pré-operatório (Fig. 8).

\section{DISCUSSÃO}

A herniação de tecido meníngeo e/ou cerebral no osso temporal é uma entidade rara e geralmente ocorre em contexto de OMC, especialmente do tipo colesteatomatosa, ou após cirurgia otológica para erradicação da mesma. A incidência de HME declinou após a introdução de antibióticos e redução da taxa de complicações intracranianas da OMC. Na era pré-antibiótica era mais frequente a ocorrência de HME como complicação de uma trepanação transmastoideia para drenagem de coleção abcedada intracraniana. ${ }^{5}$

As HME ocorrem mais frequentemente ao nível da fossa craniana média, ao nível do tegmen mastoideu e/ou timpânico, sendo raras ao nível da fossa posterior. Apesar da presença de um defeito ósseo temporal ser um requisito para a ocorrência das HME, nem todas as soluções de continuidade ósseas temporais estão associadas a HME. Esta observação baseia-se em estudos de dissecção de cadáveres, nos quais se verificou que a incidência de HME é inferior à de defeitos ósseos temporais. Assim, pressupõe-se que seja necessária a presença de um defeito ou zona de fragilidade ao nível da dura-máter para que ocorra a HME. ${ }^{1,5}$
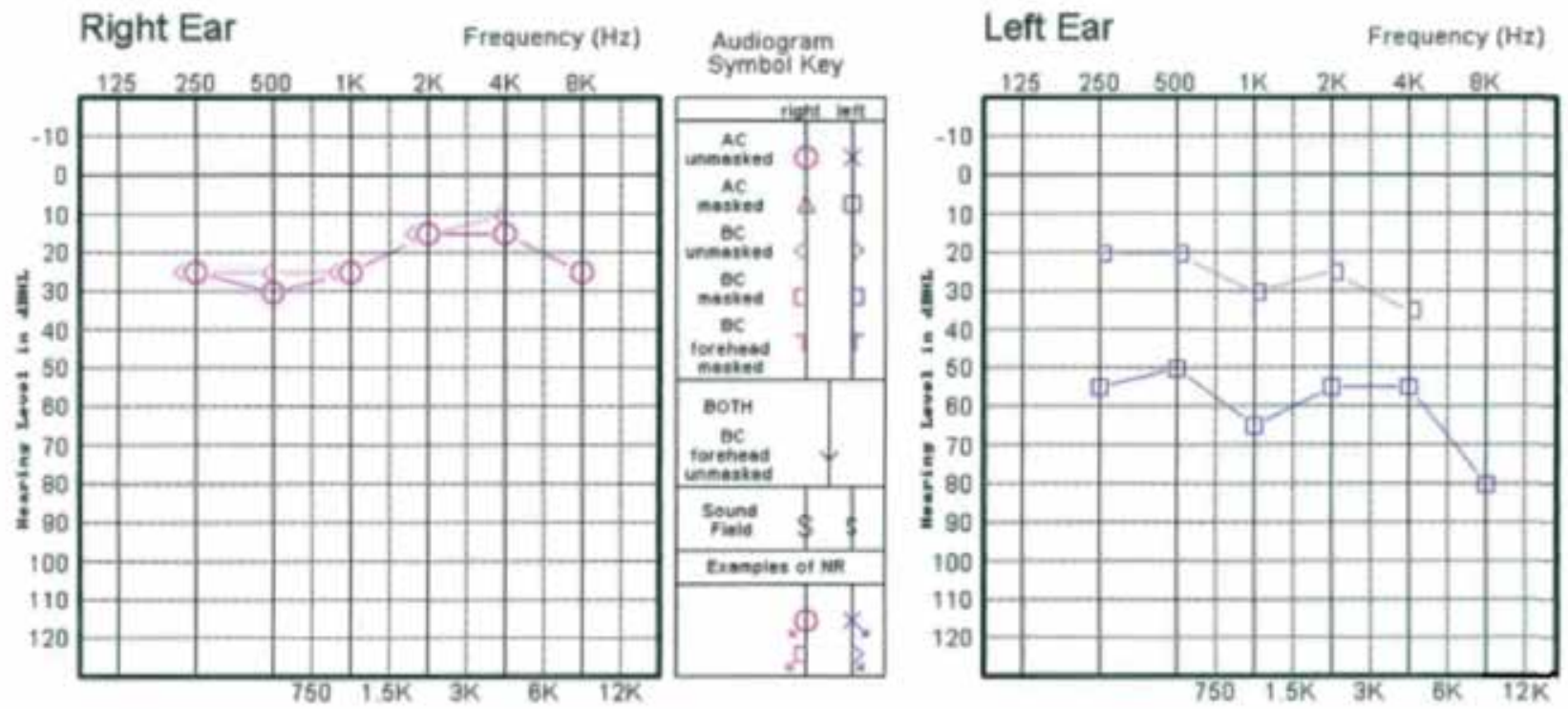

FIGURA 8. Audiograma tonal pós-operatório. 
O diagnóstico desta entidade pode representar um verdadeiro desafio para o clínico tendo em conta os achados, por vezes inespecíficos, com os quais se apresenta. O quadro de manifestações clínicas das HME dependerá da patogénese da mesma. As HME adquiridas em contexto de OMC, com ou sem colesteatoma, terão uma apresentação clínica dominada pela sintomatologia da doença otológica de base, nomeadamente: hipoacusia, otorreia, tinnitus e/ou vertigem. HME espontâneas poderão apresentar-se através de hipoacusia e plenitude aural semelhante a um quadro de otite média com efusão, ou através de otorráquia. Por vezes a manifestação de uma HME poderá ser um evento satélite como: meningite, abcesso cerebral ou epilepsia com origem em foco temporal. À otoscopia pode ser possível observar uma massa pulsátil retro-timpânica, no canal auditivo externo (CAE) ou na cavidade de mastoidectomia. ${ }^{6,7}$

A avaliação radiológica é essencial para o diagnóstico das HME. A tomografia computorizada de alta resolução (TCAR) apresenta melhor resolução óssea, sendo útil na determinação da localização, número e tamanho do defeito ósseo. No entanto, a presença de uma massa com densidade de tecidos moles no ouvido médio é um achado inespecífico, sendo impossível a distinção entre tecido cerebral herniado, colesteatoma, granuloma de colesterol ou tecido de granulação. Neste sentido, a ressonância magnética (RM), com a sua melhor definição de tecidos moles, poderá complementar o estudo por TC. As HME são isointensas com o parênquima cerebral em todas as sequências. Por outro lado, o colesteatoma é hiperintenso na ponderação T2 e o granuloma de colesterol é hiperintenso em ambas as ponderações (T1 e T2). Após administração de contraste endovenoso apenas o tecido de granulação sofre captação. ${ }^{8}$

As HME apesar de raras podem condicionar complicações fatais para o paciente, pelo que, a sua correção cirúrgica é recomendada. Na literatura estão descritos três tipos de abordagem: via transmastoideia (TM), via da fossa média (FM) e via combinada. Alguns autores consideram ainda a obliteração do ouvido médio como um método de tratamento das HME. ${ }^{9}$ Diversos fatores interferem na escolha da via de abordagem cirúrgica, nomeadamente: etiologia da HME, tamanho e localização do defeito ósseo temporal, audiometria pré-operatória, presença de OMC e presença de fístula de LCR intra-operatória. ${ }^{1,7}$

A via TM é útil nos casos de deiscências únicas, de pequenas dimensões, localizadas ao nível do tegmen mastoideu ou antri e sem atingimento da cadeia ossicular. Se a cadeia ossicular for removida poderão ser reparados defeitos ósseos localizados mais anteriormente. A via TM poderá ser igualmente utilizada nos casos de HME da fossa posterior.

A via da FM permite uma exposição ampla do pavimento da fossa craniana média, sendo útil em casos de HME de localização anterior e nos quais se pretende manter a cadeia ossicular intacta. Em casos de OMC ativa a via da FM permite a reparação da HME, isolando o ouvido médio infetado do compartimento intracraniano.

A via combinada permite a reparação de defeitos ósseos amplos a partir "de cima" através da realização de uma mini-craniotomia.

A obliteração do ouvido médio implica o encerramento em fundo-de-saco do CAE, com realização de uma petrosectomia subtotal, obliteração do orifício da trompa de Eustáquio e preenchimento da cavidade com gordura abdominal. Tendo em conta o grau de surdez resultante, esta técnica deve ser reservada para casos com hipoacusia acentuada pré-operatória e destruição extensa do ouvido médio com limitadas hipóteses de reconstrução.

Diversos materiais podem ser utilizados na reconstrução do defeito ósseo e/ou dural, nomeadamente fáscia temporalis, fáscia lata, cartilagem, pó de osso ou músculo. Poderão ser igualmente utilizados materiais sintéticos como dura-liofilizada, placa de titânio ou cristais de hidroxiapatite na reparação dos defeitos ósseos. De acordo com a literatura, a reconstrução em multicamadas é preferível à reparação em cada única, estando associada a menor taxa de recidiva. ${ }^{10}$

\section{CONCLUSÃO}

As herniações meningoencefálicas são entidades raras que requerem tratamento cirúrgico adequado de modo a prevenir complicações potencialmente fatais. No diagnóstico é essencial a informação complementar fornecida pela tomografia computorizada e ressonância magnética. $\bigcirc$ tratamento é cirúrgico, estando descritas diversas vias de abordagem, sendo fundamental a colaboração multidisciplinar entre a Otorrinolaringologia e a Neurocirurgia.

CONFLITOS DE INTERESSE: Os autores declaram a inexistência de conflitos de interesse na realização do trabalho.

FONTES DE FINANCIAMENTO: Não existiram fontes externas de financiamento para a realização deste artigo.

CONFIDENCIALIDADE DOS DADOS: Os autores declaram ter seguido os protocolos do seu centro de trabalho acerca da publicação dos dados de doentes. 
PROTEÇÃO DE PESSOAS E ANIMAIS: Os autores declaram que os procedimentos seguidos na elaboração do presente trabalho estão em conformidade com as normas das comissões de investigação clínica e de ética, bem como da declaração de Helsínquia e da Associação Médica Mundial.

\section{REFERÊNCIAS}

1. Sanna M, Paolo F, Russo A, Falcioni M. Management of meningoencephalic herniation of the temporal bone: personal experience and literature review. Laryngoscope. 2009;119:1579-85.

2. Caboche H. De la hernie cerebral dans les interventions intracraniennes dirigees contre les moyennes suppurees. Ann Mal Poriell Laryngx. 1902;28:278-94.

3. Aristegui M, Falcioni M, Saleh E, Taibah A, Russo A, Landolfi M, et al. Meningoencephalic herniation into the middle ear: a report of 27 cases. Laryngoscope. 1995;105:513-8.

4. Brown NE, Grundfast KM, Jabre A, Megerian CA, O'Malley Jr BW, Rosenberg SI. Diagnosis and management of spontaneous cerebrospinal fluid-middle ear effusion and otorrhea. Laryngoscope. 2004;114:800-5.
5. Chen DA. Dural herniation and cerebrospinal fluid leaks. In: Brackmann DE, Arriaga MA, Chelton C, editors. Otologic Surgery. $3^{\mathrm{a}}$ ed. Amsterdam: Saunders Elsevier;2010. p.245-51.

6. Sergi B, Passali CG, Picciotti PM, De Corso E, Paludetti G. Transmastoid approach to repair meningoencephalic herniation in the middle ear. Acta Otorhinolaryngol Ital. 2013;33:97101.

7. Blioskas S, Magras I, Polyzoidis S, Kouskouras K, Psillas G, Dova S, et al. Repair of temporal bone encephalocele following canal wall down mastoidectomy. Case Rep Otolaryngol. 2014:271824.

8. Bovo R, Ceruti S, Padovani R, Martini A. Temporal bone brain herniation: Imaging case of the month. Otol Neurotol. 2006;27:576-7.

9. Sanna M, Dispenza F, Flanagan S, De Stefano A, Falcioni M. Management of chronic otitis by middle ear obliteration with blind sac closure of the external auditory canal. Otol Neurotol. 2007;29:19-22.

10. Savva A, Taylor MJ, Beatty CW. Management of cerebro-spinal fluid leaks involving the temporal bone: report on $92 \mathrm{pa}$ tients. Laryngoscope. 2006;113:50-6. 